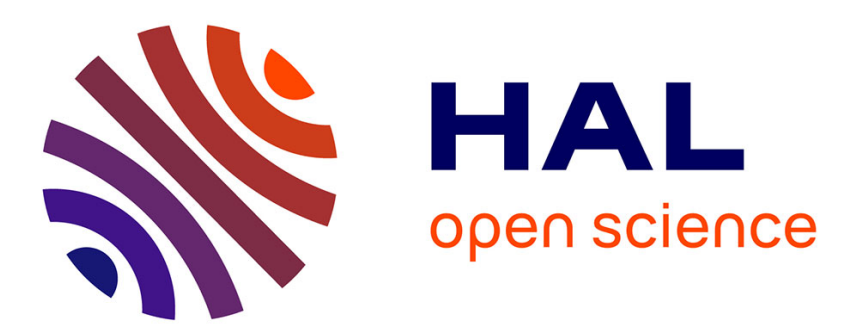

\title{
Comparing Mirrored Mutations and Active Covariance Matrix Adaptation in the IPOP-CMA-ES on the Noiseless BBOB Testbed
}

Dimo Brockhoff, Anne Auger, Nikolaus Hansen

\section{- To cite this version:}

Dimo Brockhoff, Anne Auger, Nikolaus Hansen. Comparing Mirrored Mutations and Active Covariance Matrix Adaptation in the IPOP-CMA-ES on the Noiseless BBOB Testbed. GECCO Companion '12, Jul 2012, Philadelphia, PA, United States. pp.297-303, 10.1145/2330784.2330827 • hal-00746120

HAL Id: hal-00746120

https://hal.inria.fr/hal-00746120

Submitted on 27 Oct 2012

HAL is a multi-disciplinary open access archive for the deposit and dissemination of scientific research documents, whether they are published or not. The documents may come from teaching and research institutions in France or abroad, or from public or private research centers.
L'archive ouverte pluridisciplinaire HAL, est destinée au dépôt et à la diffusion de documents scientifiques de niveau recherche, publiés ou non, émanant des établissements d'enseignement et de recherche français ou étrangers, des laboratoires publics ou privés. 


\section{Comparing Mirrored Mutations and Active Covariance Matrix Adaptation in the IPOP-CMA-ES on the Noiseless BBOB Testbed}

\author{
Dimo Brockhoff \\ INRIA Lille - Nord Europe \\ Dolphin team \\ 59650 Villeneuve d'Ascq \\ France \\ dimo.brockhoff@inria.fr
}

\author{
Anne Auger \\ Projet TAO, INRIA \\ Saclay-lle-de-France \\ LRI, Bât 490, Univ. Paris-Sud \\ 91405 Orsay Cedex, France \\ anne.auger@inria.fr
}

\author{
Nikolaus Hansen \\ Projet TAO, INRIA \\ Saclay-lle-de-France \\ LRI, Bât 490, Univ. Paris-Sud \\ 91405 Orsay Cedex, France \\ nikolaus.hansen@inria.fr
}

\begin{abstract}
This paper investigates two variants of the well-known Covariance Matrix Adaptation Evolution Strategy (CMA-ES). Active covariance matrix adaptation allows for negative weights in the covariance matrix update rule such that "bad" steps are (actively) taken into account when updating the covariance matrix of the sample distribution. On the other hand, mirrored mutations via selective mirroring also take the "bad" steps into account. In this case, they are first evaluated when taken in the opposite direction (mirrored) and then considered for regular selection. In this study, we investigate the difference between the performance of the two variants empirically on the noiseless BBOB testbed. The CMA-ES with selectively mirrored mutations only outperforms the active CMA-ES on the sphere function while the active variant statistically significantly outperforms mirrored mutations on 10 of 24 functions in several dimensions.
\end{abstract}

\section{Categories and Subject Descriptors}

G.1.6 [Numerical Analysis]: Optimization-global optimization, unconstrained optimization; F.2.1 [Analysis of Algorithms and Problem Complexity]: Numerical Algorithms and Problems

\section{General Terms}

Algorithms

\section{Keywords}

Benchmarking, Black-box optimization

\section{INTRODUCTION}

The covariance matrix adaptation evolution strategy (CMAES) is considered as a standard method for stochastic optimization in continuous domain. More recently, mirrored

(C)ACM, 2012. This is the authors' version of the work. It is posted here by permission of ACM for your personal use. Not for redistribution. The definitive version was published at GECCO Companion '12, July 7-11, 2012, Philadelphia, PA, USA. http://doi.acm.org/10.1145/2330784.2330827 mutations for evolution strategies have been introduced and theoretically investigated in a number of papers $[4,1,2]$. In evolution strategies with weighted recombination and only positive recombination weights, mirrored mutations can improve the possible progress rate on the sphere function by about $56 \%$ [2]. Carefully implemented, mirrored mutations retain unbiasedness. In this paper, we use these mirrored mutations with CMA-ES and compare the performance with active covariance matrix adaptation [9]. The latter is also based on the idea to use bad examples, but in the context of covariance matrix adaptation. Active CMA-ES has shown to consistently outperform the standard CMA-ES variant on the BBOB testbed [8]. In this paper, both algorithms are compared using restarts with increasing population size (IPOP-CMA-ES, [3]).

\section{THE CONSIDERED ALGORITHM VARI- ANTS}

Mirrored mutations together with selective mirroring has been implemented according to [2] into the CMA-ES. In particular, selective mirroring with $\lambda_{\mathrm{m}}=\left\lfloor 0.5+0.159 \lambda_{\text {iid }}\right\rfloor$ is used together with the standard recombination weights [2]. We denote the corresponding algorithm by $\mathrm{CMA}_{\mathrm{m}}$.

Active covariance matrix adaptation [9] has been implemented as in [8]. This algorithm will be referred to as $\mathrm{CMA}_{\mathrm{a}}$. As a baseline algorithm, we also show results for the IPOP-CMA-ES that does neither use the active covariance matrix adaptation nor mirrored mutations. All three algorithms use the same parameter settings that are slightly different from those in [8]. They were restarted up to 9 times with the population size doubling each time and up to the maximal number of overall function evaluations of $2 \cdot 10^{5} \cdot D$ with $D$ the problem dimension. For the experiments, we used version 3.54.beta.mirrors of the MATLAB implementation which can be downloaded from http: //canadafrance.gforge.inria.fr/mirroring/.

\section{TIMING EXPERIMENTS}

In order to see the dependency of the algorithms on the problem dimension, the requested BBOB'2012 timing experiment has been performed for the original IPOP-CMAES and the variants $\mathrm{CMA}_{\mathrm{m}}$ with mirrored mutations and $\mathrm{CMA}_{\mathrm{a}}$ with active covariance matrix adaptation on an Intel Core2 Duo T9600 laptop with 2.80GHz, 4.0GB of RAM, and 
MATLAB R2008b on Windows Vista SP2. The algorithms have been restarted for up to $2 \cdot 10^{5} \mathrm{~N}$ function evaluations until 30 seconds have passed. The per-function-evaluationruntimes were $18,14,9.8,5.5,4.2,4.3,6.6$ times $10^{-4}$ seconds for the IPOP-CMA-ES, 23, 16, 9.3, 5.3, 4.4, 4.9, 6.2 times $10^{-4}$ seconds for the $\mathrm{CMA}_{\mathrm{m}}$, and 25, 18, 13, 7.9, 5.5, 5.5, and 7.4 times $10^{-4}$ seconds for the $\mathrm{CMA}_{\mathrm{a}}$ in $2,3,5,10$, 20, 40, and 80 dimensions respectively.

\section{RESULTS}

Results from experiments according to [6] on the benchmark functions given in [5, 7] are presented in Figures 1, 2 and 3 and in Tables 1 and 2 . The expected running time (ERT), used in the figures and table, depends on a given target function value, $f_{\mathrm{t}}=f_{\mathrm{opt}}+\Delta f$, and is computed over all relevant trials as the number of function evaluations executed during each trial while the best function value did not reach $f_{\mathrm{t}}$, summed over all trials and divided by the number of trials that actually reached $f_{\mathrm{t}}[6,10]$. Statistical significance is tested with the rank-sum test for a given target $\Delta f_{\mathrm{t}}$ $\left(10^{-8}\right.$ as in Figure 1) using, for each trial, either the number of needed function evaluations to reach $\Delta f_{\mathrm{t}}$ (inverted and multiplied by -1 ), or, if the target was not reached, the best $\Delta f$-value achieved, measured only up to the smallest number of overall function evaluations for any unsuccessful trial under consideration.

A significant improvement due to mirrored mutations can be observed on the sphere function only. Mirrored mutations speed up CMA-ES by about $35 \%$ in this case. Otherwise, no statistically significant effect of mirrored mutations is observed within the given experimental setup. In particular, mirrored mutations also do not lead to a failure where the original algorithm succeeds. As observed already before, active CMA-ES improves the performance on many ill-conditioned unimodal problems, usually also by less than a factor of two.

\section{REFERENCES}

[1] A. Auger, D. Brockhoff, and N. Hansen. Analyzing the Impact of Mirrored Sampling and Sequential Selection in Elitist Evolution Strategies. In Foundations of Genetic Algorithms (FOGA 2011), pages 127-138. ACM, 2011.
[2] A. Auger, D. Brockhoff, and N. Hansen. Mirrored Sampling in Evolution Strategies With Weighted Recombination. In Genetic and Evolutionary Computation Conference (GECCO 2011), pages 861-868. ACM, 2011.

[3] A. Auger and N. Hansen. A Restart CMA Evolution Strategy With Increasing Population Size. In Congress on Evolutionary Computation (CEC 2005), volume 2, pages 1769-1776. IEEE Press, 2005.

[4] D. Brockhoff, A. Auger, N. Hansen, D. V. Arnold, and T. Hohm. Mirrored Sampling and Sequential Selection for Evolution Strategies. In Conference on Parallel Problem Solving from Nature (PPSN XI), pages 11-21. Springer, 2010.

[5] S. Finck, N. Hansen, R. Ros, and A. Auger. Real-parameter black-box optimization benchmarking 2009: Presentation of the noiseless functions. Technical Report 2009/20, Research Center PPE, 2009. Updated February 2010.

[6] N. Hansen, A. Auger, S. Finck, and R. Ros. Real-parameter black-box optimization benchmarking 2012: Experimental setup. Technical report, INRIA, 2012.

[7] N. Hansen, S. Finck, R. Ros, and A. Auger. Real-parameter black-box optimization benchmarking 2009: Noiseless functions definitions. Technical Report RR-6829, INRIA, 2009. Updated February 2010.

[8] N. Hansen and R. Ros. Benchmarking a weighted negative covariance matrix update on the BBOB-2010 noiseless testbed. In Genetic and Evolutionary Computation Conference (GECCO 2010), pages 1673-1680, New York, NY, USA, 2010. ACM.

[9] G. Jastrebski and D. Arnold. Improving evolution strategies through active covariance matrix adaptation. In IEEE Congress on Evolutionary Computation (CEC 2006), pages 2814-2821, 2006.

[10] K. Price. Differential evolution vs. the functions of the second. In Proceedings of the IEEE International Congress on Evolutionary Computation (ICEO), pages 153-157, 1997. 

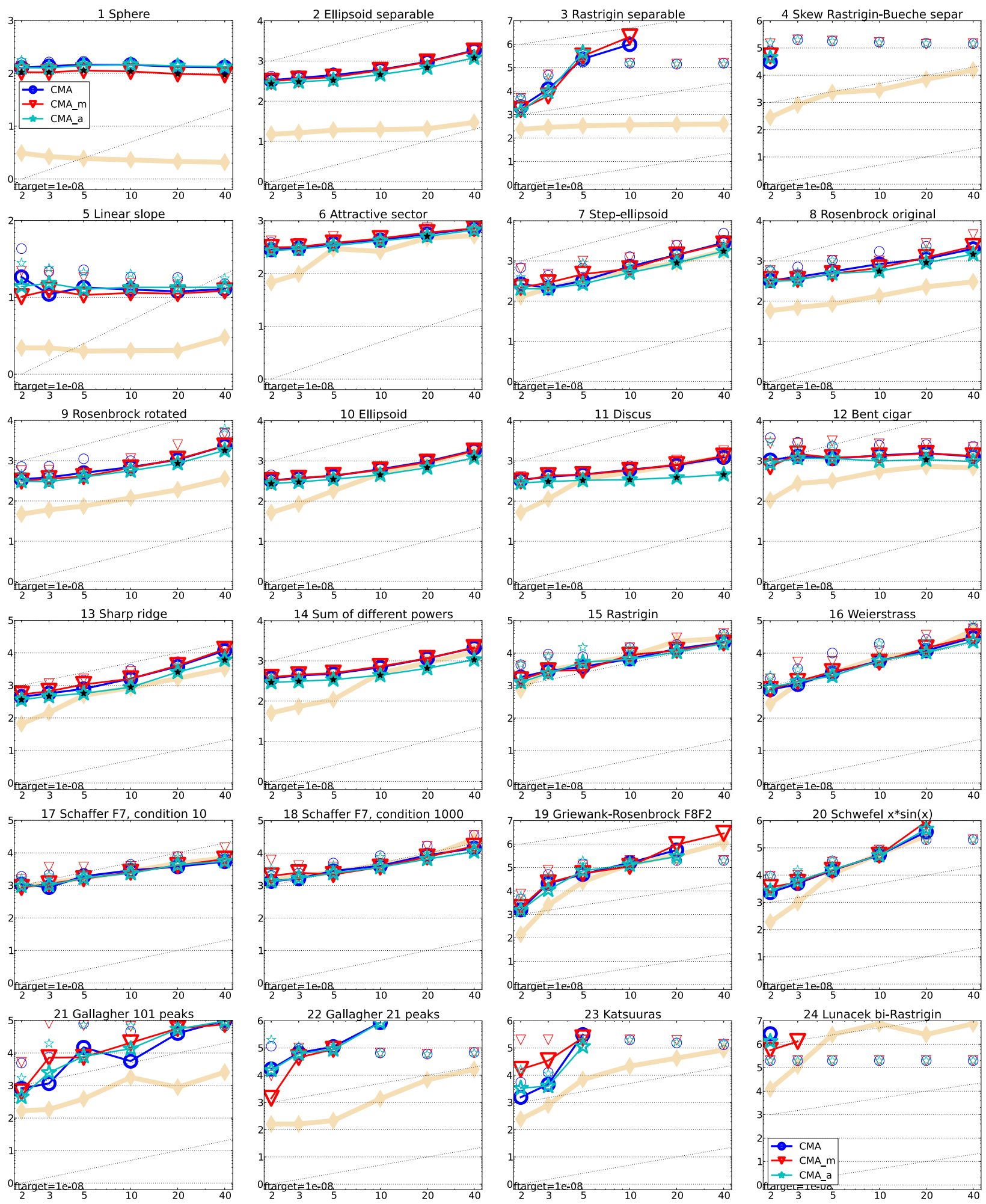

Figure 1: Expected running time (ERT in number of $f$-evaluations) divided by dimension for target function value $10^{-8}$ as $\log _{10}$ values versus dimension. Different symbols correspond to different algorithms given in the legend of $f_{1}$ and $f_{24}$. Light symbols give the maximum number of function evaluations from the longest trial divided by dimension. Horizontal lines give linear scaling, slanted dotted lines give quadratic scaling. Black stars indicate statistically better result compared to all other algorithms with $p<0.01$ and Bonferroni

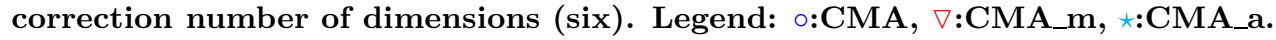



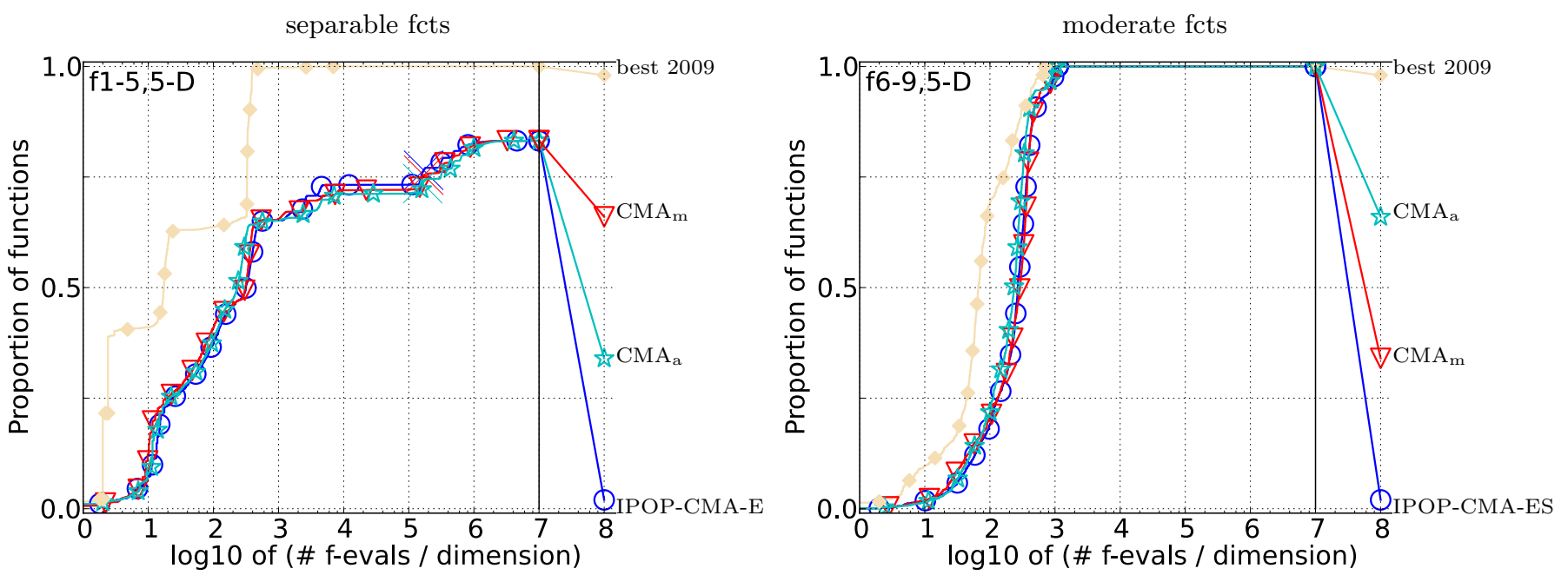

ill-conditioned fcts
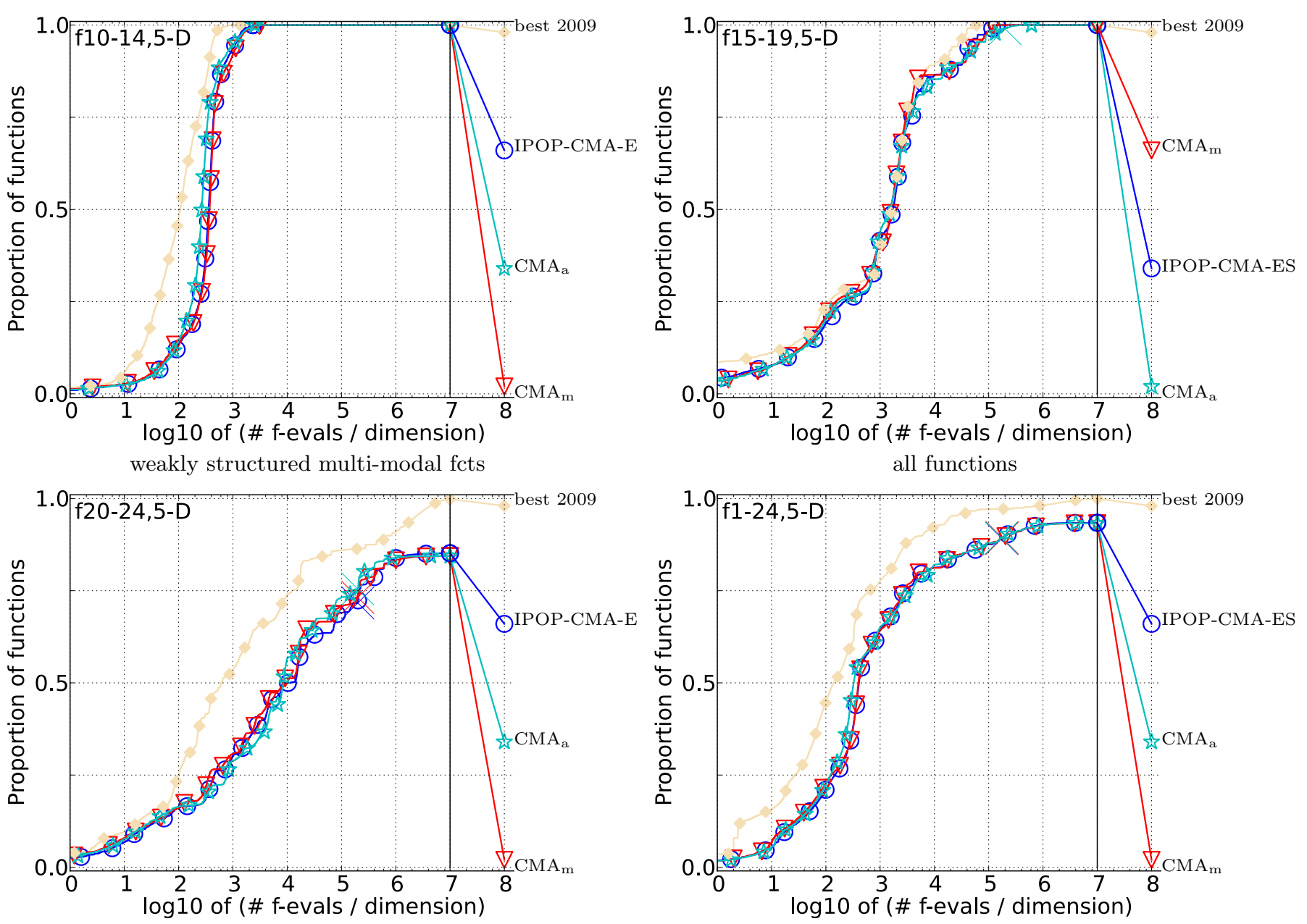

Figure 2: Bootstrapped empirical cumulative distribution of the number of objective function evaluations divided by dimension (FEvals/D) for 50 targets in $10^{[-8 . .2]}$ for all functions and subgroups in 5-D. The "best 2009" line corresponds to the best ERT observed during BBOB 2009 for each single target. 

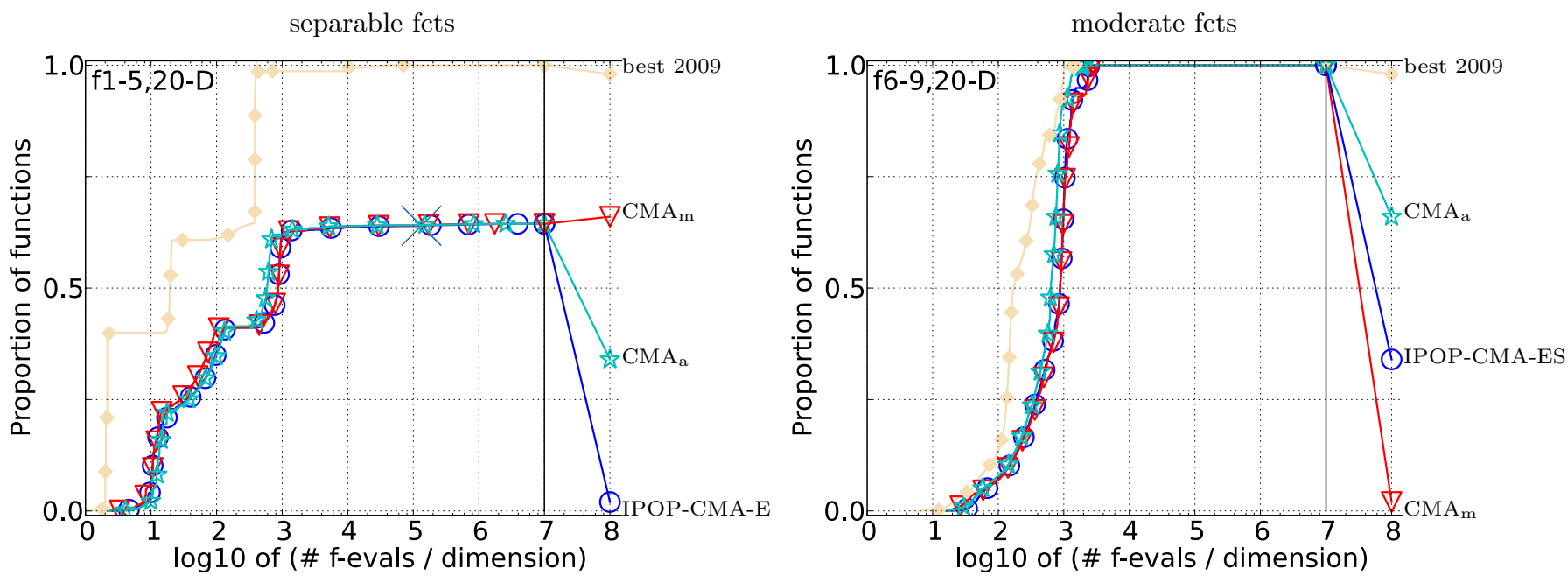

ill-conditioned fcts
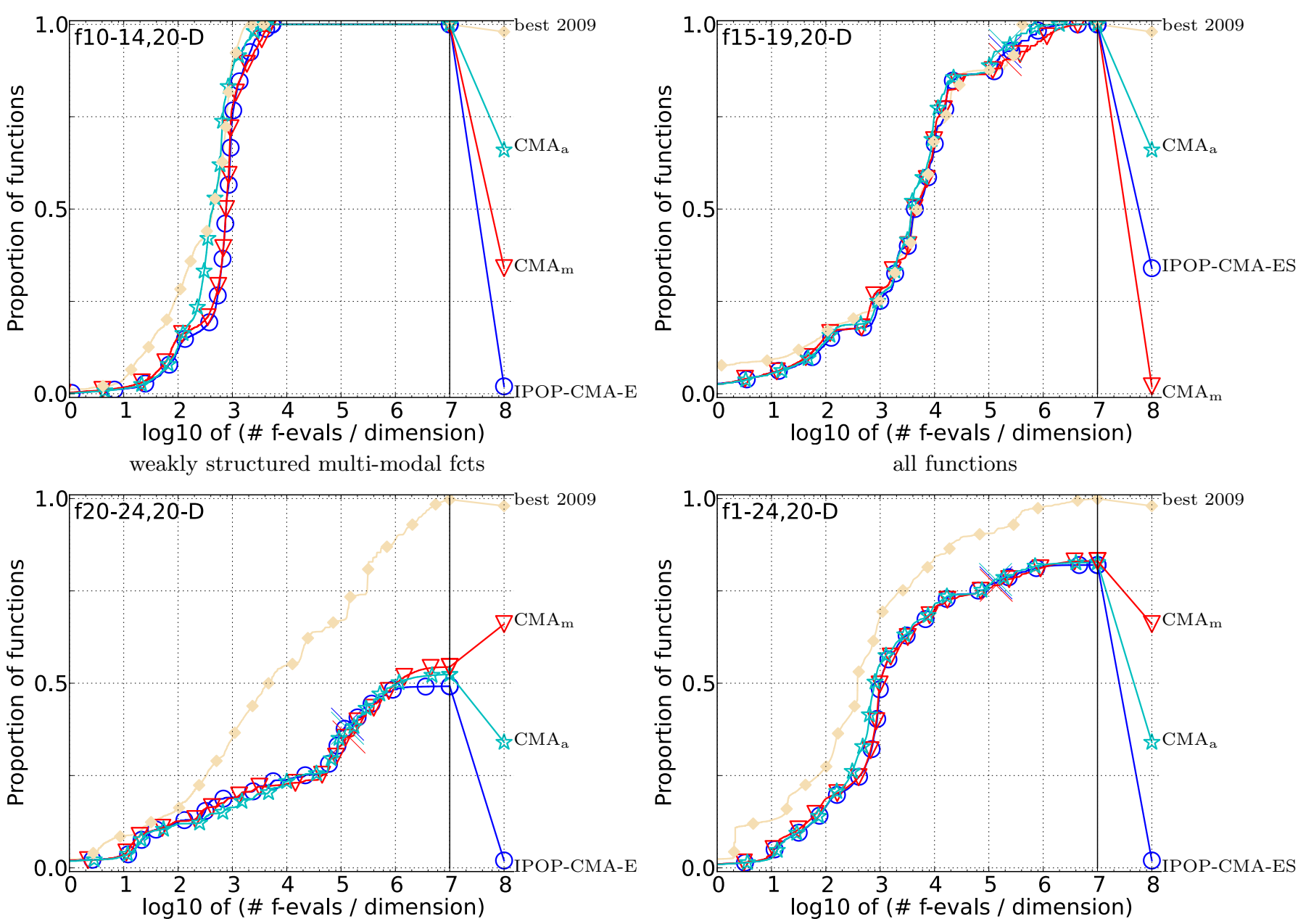

Figure 3: Bootstrapped empirical cumulative distribution of the number of objective function evaluations divided by dimension (FEvals/D) for 50 targets in $10^{[-8.2]}$ for all functions and subgroups in 20-D. The "best 2009" line corresponds to the best ERT observed during BBOB 2009 for each single target. 


\begin{tabular}{|c|c|c|c|c|c|c|c|c|c|c|c|c|c|c|c|}
\hline$\Delta f_{\text {opt }}$ & $1 \mathrm{e} 1$ & $1 \mathrm{e} 0$ & $1 \mathrm{e}-1$ & $1 \mathrm{e}-3$ & $1 e-5$ & $1 \mathrm{e}-7$ & \#succ & $\Delta f_{\text {opt }}$ & $1 \mathrm{e} 1$ & $1 \mathrm{e} 0$ & $1 \mathrm{e}-1$ & $1 e-3$ & $1 e-5$ & $1 \mathrm{e}-7$ & \#succ \\
\hline f1 & 11 & 12 & 12 & 12 & 12 & 12 & $15 / 15$ & f13 & 132 & 195 & 250 & 1310 & 1752 & 2255 & $15 / 15$ \\
\hline $\mathrm{CMA}$ & $2.6(3)$ & $9.3(4)$ & $15(4)$ & $28(4)$ & $40(5)$ & $54(6)$ & $15 / 15$ & CMA & $3.3(2)$ & $5.3(2)$ & $5.5(2)$ & $1.4(0.3)$ & $1.6(0.3)$ & $1.5(0.3)$ & $15 / 15$ \\
\hline mir & $2.8(2)$ & $7.6(2)$ & $12(3)$ & $22(4)$ & $30(5) \star 3$ & $41(5)^{\star 2}$ & $15 / 15$ & $\mathrm{mir}$ & $4.0(3)$ & $5.0(2)$ & $4.7(2)$ & $1.7(0.7)$ & $1.8(0.8)$ & $2.0(0.8)$ & $15 / 15$ \\
\hline act & $2.5(2)$ & $8.1(4)$ & $15(4)$ & $25(5)$ & $38(4)$ & $51(8)$ & $15 / 15$ & act & $2.9(0.7)$ & $4.1(2)$ & $4.5(1)$ & $1.2(0.2)$ & $1.2(0.1)^{\star}$ & $1.2(0.1)^{\star 3}$ & $15 / 15$ \\
\hline$\Delta f_{\text {opt }}$ & $1 \mathrm{e} 1$ & $1 \mathrm{e} 0$ & $1 \mathrm{e}-1$ & $1 \mathrm{e}-3$ & $1 \mathrm{e}-5$ & $1 \mathrm{e}-7$ & \#succ & $\Delta f_{\text {opt }}$ & $1 \mathrm{e} 1$ & $1 \mathrm{e} 0$ & $1 \mathrm{e}-1$ & $1 \mathrm{e}-3$ & $1 e-5$ & $1 \mathrm{e}-7$ & \#succ \\
\hline f2 & 83 & 87 & 88 & 90 & 92 & 94 & $15 / 15$ & f14 & 10 & 41 & 58 & 139 & 251 & 476 & $15 / 15$ \\
\hline CMA & $14(4)$ & $16(4)$ & $17(4)$ & $20(3)$ & $22(3)$ & $23(2)$ & $15 / 15$ & CMA & $2.3(3)$ & $2.8(0.9)$ & $3.5(1)$ & $4.2(1)$ & $5.4(0.5)$ & 4.4( & $15 / 15$ \\
\hline mir & $13(5)$ & $16(4)$ & $17(4)$ & $19(2)$ & $20(1)$ & $21(1.0)$ & $15 / 15$ & mir & $1.6(2)$ & $2.8(1)$ & $3.2(2)$ & $4.1(1.0)$ & $5.4(1)$ & $4.4(0.6)$ & $15 / 15$ \\
\hline act & $10(3)$ & $12(2)$ & $13(2)$ & $15(1)^{\star 3}$ & $16(2)^{\star 4}$ & $17(1)^{\star 4}$ & $15 / 15$ & act & $2.5(3)$ & $2.7(1)$ & $3.5(1)$ & $4.0(0.7)$ & $3.9(0.4) \star 2$ & $3.1(0.4)^{\star 4}$ & $15 / 15$ \\
\hline$\Delta f_{\text {opt }}$ & $1 \mathrm{e} 1$ & $1 \mathrm{e} 0$ & $1 e-1$ & $1 \mathrm{e}-3$ & $1 \mathrm{e}-5$ & $1 \mathrm{e}-7$ & \#succ & $\Delta f_{\text {opt }}$ & $1 \mathrm{e} 1$ & $1 \mathrm{e} 0$ & $1 \mathrm{e}-1$ & $1 \mathrm{e}-3$ & $1 e-5$ & $1 \mathrm{e}-7$ & \#succ \\
\hline $\mathrm{f3}$ & 716 & 1622 & 1637 & 1646 & 1650 & 1654 & $15 / 15$ & f15 & 511 & 9310 & 19369 & 20073 & 20769 & 21359 & $14 / 15$ \\
\hline $\mathrm{CMA}$ & $1.4(2)$ & $7.9(6)$ & $\mathbf{7 1 8}(939)$ & $\mathbf{7 1 5}(936)$ & $\mathbf{7 1 3}(932)$ & $712(945)$ & $6 / 15$ & CMA & $1.6(2)$ & $0.74(0.5)$ & $0.86(0.6)$ & 0.86 & $0.6)$ & & $15 / 15$ \\
\hline $\mathrm{mir}$ & $1.1(1)$ & $\mathbf{7 . 0}(10)$ & $959(1347)$ & $955(1367)$ & $953(1162)$ & $951(1218)$ & $5 / 15$ & $\operatorname{mir}$ & $1.8(2)$ & $0.74(0.6)$ & $0.66(0.4)$ & $0.67(0.4)$ & $0.67(0.3)$ & 0.68 & $15 / 15$ \\
\hline act & $\mathbf{0 . 9 1}(0.6) 30$ & $30(7)$ & $333(1489)$ & $326(1644)$ & $1323(1663)$ & $1321(1644)$ & $4 / 15$ & act & $1.5(2)$ & $1.1(0.7)$ & $1.2(0.6)$ & $1.2(0.7)$ & $1.2(0.6)$ & $1.2(0.6)$ & $15 / 15$ \\
\hline$\Delta f_{\text {opt }}$ & $1 \mathrm{e} 1$ & $1 \mathrm{e} 0$ & $1 \mathrm{e}-1$ & $1 \mathrm{e}-3$ & $1 \mathrm{e}-5$ & $1 \mathrm{e}-7$ & \#succ & $\Delta f_{\text {opt }}$ & $1 \mathrm{e} 1$ & $1 \mathrm{e} 0$ & $1 \mathrm{e}-1$ & $1 e-3$ & $1 e-5$ & $1 e-7$ & \#succ \\
\hline$f 4$ & 809 & 1633 & 1688 & 1817 & 1886 & 1903 & $15 / 15$ & f16 & 120 & 612 & 2662 & 10449 & 11644 & 12095 & $15 / 15$ \\
\hline CMA & $2.7(3)$ & $\infty$ & $\infty$ & $\infty$ & $\infty$ & $\infty 9 e 5$ & $0 / 15$ & CMA & $2.3(2)$ & & & & & & $15 / 15$ \\
\hline ir & $2.9(3)$ & $\infty$ & $\infty$ & $\infty$ & $\infty$ & $\infty 9 e 5$ & $0 / 15$ & $\operatorname{mir}$ & $2.9(4)$ & & & & & & $15 / 15$ \\
\hline act & $1.7(2)$ & $\infty$ & $\infty$ & $\infty$ & $\infty$ & $\infty 9 e 5$ & $0 / 15$ & act & $1.7(1)$ & $2.8(3)$ & $2.2(2)$ & $0.84(0.6)$ & $0.80(0.5)$ & $\mathbf{0 . 8 0}(0.5)$ & $\mid 15 / 15$ \\
\hline$\Delta f_{\text {opt }}$ & $1 \mathrm{e} 1$ & $1 \mathrm{e} 0$ & $1 \mathrm{e}-1$ & $1 \mathrm{e}-3$ & $1 e-5$ & $1 \mathrm{e}-7$ & \#succ & $\Delta f_{\text {opt }}$ & $1 \mathrm{e} 1$ & $1 \mathrm{e} 0$ & $1 \mathrm{e}-1$ & $1 \mathrm{e}-3$ & $1 \mathrm{e}-5$ & $1 \mathrm{e}-7$ & \#succ \\
\hline f5 & 10 & 10 & 10 & 10 & 10 & 10 & $15 / 15$ & f17 & 5.2 & 215 & 899 & 3669 & 6351 & 7934 & $15 / 15$ \\
\hline CMA & $4.4(2)$ & $6.5(2)$ & $7(2)$ & $6.7(2)$ & $7(2)$ & $6.7(2)$ & $15 / 15$ & CMA & $1.8(2)$ & $2(0.3)$ & $0.93(2)$ & $0.89(0.6)$ & 1. & & $15 / 15$ \\
\hline ir & $3.9(2$ & $5.1(2$ & $3(1)$ & $.4(1)$ & 5 & 5.4( & $15 / 15$ & $\operatorname{mir}$ & 3.4 & & & & & & $15 / 15$ \\
\hline act & $4.2(2)$ & $6.0(2)$ & $6.3(2)$ & $6.4(2)$ & $6.4(2)$ & $6.4(2)$ & $15 / 15$ & act & $2.6(2)$ & $1.3(0.4)$ & $0.77(1.0)$ & $0.89(0.5)$ & $0.81(0.3)$ & $1.0(0.4)$ & $15 / 15$ \\
\hline$\Delta f_{\text {opt }}$ & $1 \mathrm{e} 1$ & $1 \mathrm{e} 0$ & $1 \mathrm{e}-1$ & $1 e-3$ & $1 e-5$ & $1 e-7$ & \#succ & $\Delta f_{\text {opt }}$ & $1 \mathrm{e} 1$ & $1 \mathrm{e} 0$ & $1 \mathrm{e}-1$ & $1 e-3$ & $1 e-5$ & $1 e-7$ & \#succ \\
\hline$f 6$ & 114 & 214 & 281 & 580 & 1038 & 1332 & $15 / 15$ & f18 & 103 & 378 & 3968 & 9280 & & & $15 / 15$ \\
\hline CMA & $2.5(0.9)$ & $2.2(0.4)$ & $(0.3)$ & $(0.2)$ & & .1) & $15 / 15$ & CMA & $1.2(0$. & 1.6 & & 0.5 & & & $15 / 15$ \\
\hline ir & $2.2(1$ & $2.0(0$ & 2.1 & & & & $15 / 15$ & $\operatorname{mir}$ & 0.9 & & $0.53(0.6)$ & & & & $15 / 15$ \\
\hline act & $2.0(0.6)$ & $1.9(0.4)$ & $2.0(0.3)$ & $1.5(0.2)$ & $1.2(0.1)$ & $1.1(0.1)$ & $15 / 15$ & act & $0.82(0.3)$ & $1.7(0.3)$ & $0.44(0.5)$ & $\mathbf{0 . 6 6}(0.3)$ & $0.76(0.3)$ & $0.94(0.6)$ & $15 / 15$ \\
\hline$\Delta f_{\text {opt }}$ & $1 \mathrm{e} 1$ & $1 \mathrm{e} 0$ & $1 e-1$ & $1 \mathrm{e}-3$ & $1 e-5$ & $1 \mathrm{e}-7$ & \#succ & $\Delta f_{\text {opt }}$ & $1 \mathrm{e} 1$ & $1 \mathrm{e} 0$ & $1 \mathrm{e}-1$ & $1 \mathrm{e}-3$ & $1 \mathrm{e}-5$ & $1 \mathrm{e}-7$ & \#succ \\
\hline $\mathrm{f7}$ & 24 & 32 & 1171 & 1572 & 1572 & 1597 & $15 / 15$ & f19 & 1 & 1 & 242 & $1.2 \mathrm{e} 5$ & & $1.2 \mathrm{e} 5$ & $15 / 15$ \\
\hline CMA & $4.7(3)$ & $1.5(1)$ & $88(0.4)$ & $0.92(0.7)$ & $0.92(0.7)$ & $(0.7)$ & $15 / 15$ & CMA & $15(14)$ & 17 & 572( & & & & $15 / 15$ \\
\hline ir & $3.9(2)$ & $1.1(1.0)$ & $\int(1)$ & $1.3(1)$ & $1.3(1)$ & $1.4(1)$ & $15 / 15$ & $\mathrm{mir}$ & & & & & & & \\
\hline act & 7.3(3) & $1.1(1)$ & $0.88(0.6)$ & $0.77(0.5)$ & $0.77(0.5)$ & $0.79(0.5)$ & $15 / 15$ & act & $24(10)$ & $6888(1525)$ & $462(416)$ & $3.0(3)$ & $3.0(3)$ & $3.0(3)$ & $14 / 15$ \\
\hline$\Delta f_{\text {opt }}$ & $1 \mathrm{e} 1$ & $1 \mathrm{e} 0$ & $1 \mathrm{e}-1$ & $1 \mathrm{e}-3$ & $1 e-5$ & $1 \mathrm{e}-7$ & \#succ & $\Delta f_{\text {opt }}$ & $1 \mathrm{e} 1$ & $1 \mathrm{e} 0$ & $1 \mathrm{e}-1$ & $1 \mathrm{e}-3$ & $1 e-5$ & $1 \mathrm{e}-7$ & \#succ \\
\hline $\mathrm{f8}$ & 73 & 27 & & & & 422 & $15 / 15$ & f20 & 16 & 851 & & 54470 & & & $14 / 15$ \\
\hline $\mathrm{CMA}$ & $3.4(1.0)$ & & & & $0(4)$ & & $15 / 15$ & CMA & $3.7(2)$ & & & & & & $15 / 15$ \\
\hline ir & $2.6(0$ & $4.2(5)$ & & $2(3)$ & $4(3)$ & 5.6( & $15 / 15$ & $\mathrm{mir}$ & 3.0( & & & & & & \\
\hline act & $2.7(1.0)$ & $4.5(5)$ & $4.9(5)$ & $5.1(4)$ & $5.3(4)$ & $5.5(4)$ & $15 / 15$ & act & $2.5(2)$ & $9.1(3)$ & $1.7(1)$ & $1.4(1)$ & $1.4(1)$ & $1.4(1)$ & $15 / 15$ \\
\hline$\Delta f_{\text {opt }}$ & $1 \mathrm{e} 1$ & $1 \mathrm{e} 0$ & $1 \mathrm{e}-1$ & $1 \mathrm{e}-3$ & $1 \mathrm{e}-5$ & $1 \mathrm{e}-7$ & \#succ & $\Delta f_{\text {opt }}$ & $1 \mathrm{e} 1$ & $1 \mathrm{e} 0$ & $1 \mathrm{e}-1$ & $1 \mathrm{e}-3$ & $1 e-5$ & $1 \mathrm{e}-7$ & \#succ \\
\hline f9 & 35 & $\overline{12}$ & & & & 36 & $15 / 15$ & f21 & 41 & 1157 & 1674 & 1705 & & & $14 / 15$ \\
\hline CMA & $7.0(3)$ & 9 & & & & & $15 / 15$ & CMA & $6.6(16)$ & & & & & & \\
\hline & $5.4(4$ & & & & & & $15 / 15$ & mir & 1.4 & & $21(20)$ & 21 & & & $14 /$ \\
\hline act & $6.1(2)$ & $6.5(2)$ & $\mathbf{5 . 9}(1)$ & $5.2(1)$ & $\mathbf{5 . 2}(1.0)$ & $\mathbf{5 . 2}(0.9)$ & $15 / 15$ & act & $1.9(1)$ & $28(14)$ & $23(20)$ & $23(21)$ & $23(21)$ & $22(22)$ & $14 / 15$ \\
\hline$\Delta f_{\text {opt }}$ & $1 \mathrm{e} 1$ & $1 \mathrm{e} 0$ & $1 \mathrm{e}-1$ & $1 \mathrm{e}-3$ & $1 e-5$ & $1 \mathrm{e}-7$ & \#succ & $\Delta f_{\text {opt }}$ & $1 \mathrm{e} 1$ & $1 \mathrm{e} 0$ & $1 e-1$ & $1 \mathrm{e}-3$ & $1 e-5$ & $1 \mathrm{e}-7$ & \#succ \\
\hline f10 & 349 & & & & & & $15 / 15$ & $\frac{- \text { opt }}{\text { f22 }}$ & \begin{tabular}{|l|l|}
71 & \\
\end{tabular} & 386 & 938 & & & & $14 / 15$ \\
\hline CMA & $2.8(1)$ & 2 & & & & & $15 / 15$ & CMA & & & & & & & $6 / 15$ \\
\hline mir & $3.9(0.9)$ & $3.2(0.7)$ & $.0(0.3)$ & $3.0(0.3)$ & $2.4(0.2)$ & $2.4(0.2)$ & $15 / 15$ & $\mathrm{mir}$ & $12(2$ & $17(2$ & $\mathbf{1 4 4}(2$ & 444 & & & $7 / 15$ \\
\hline act & $2.6(0.8)$ & $2.2(0.4)$ & $2.1(0.2)$ & $2.2(0.2) \star 3$ & $31.8(0.2)^{\star 3}$ & $1.9(0.2)^{\star 3}$ & $15 / 15$ & act & $15(24)$ & $87(30)$ & $379(466)$ & $433(559)$ & $421(552)$ & $411(522)$ & $7 / 15$ \\
\hline$\Delta f_{\text {opt }}$ & $1 \mathrm{e} 1$ & $1 \mathrm{e} 0$ & $1 e-1$ & $1 e-3$ & $1 e-5$ & $1 \mathrm{e}-7$ & \#succ & $\Delta f_{\text {opt }}$ & $1 \mathrm{e} 1$ & $1 \mathrm{e} 0$ & $1 e-1$ & $1 \mathrm{e}-3$ & $1 e-5$ & $1 \mathrm{e}-7$ & \#succ \\
\hline f11 & 143 & 202 & 763 & 1177 & 1467 & 1673 & $15 / 15$ & f23 & 3.0 & 518 & 14249 & 31654 & 33030 & & $15 / 15$ \\
\hline MA & $8.7(2)$ & $7.6(1)$ & & & & & $15 / 15$ & CMA & & & & & & & $6 / 15$ \\
\hline mir & $8.4(3)$ & $7.8(1)$ & $2.3(0.3)$ & $1.7(0.2)$ & $1.4(0.2)$ & $1.3(0.1)$ & $15 / 15$ & $\operatorname{mir}$ & & & & & & & $7 / 15$ \\
\hline act & $5.2(1.0) \star 2$ & $4.6(0.7)^{\star 4}$ & $41.4(0.2)^{\star} 4$ & $1.1(0.1)^{\star 4}$ & ${ }^{4} 0.95(0.1)^{\star 4}$ & $40.93(0.1) \star 4$ & $15 / 15$ & act & $2.4(3)$ & $29(17)$ & $39(71)$ & $18(32)$ & $17(30)$ & $\mathbf{1 7}(16)$ & $10 / 15$ \\
\hline$\Delta f_{\text {opt }}$ & $1 \mathrm{e} 1$ & $1 \mathrm{e} 0$ & & & & $1 \mathrm{e}-7$ & \#succ & $\Delta f_{\text {opt }}$ & $1 \mathrm{e} 1$ & $1 \mathrm{e} 0$ & $1 \mathrm{e}-1$ & $1 \mathrm{e}-3$ & $1 \mathrm{e}-5$ & $1 \mathrm{e}-7$ & \#succ \\
\hline f12 & 108 & & & & & 1494 & $15 / 15$ & $\mathrm{f24}$ & 1622 & $2.2 \mathrm{e} 5$ & $6.4 \mathrm{e} 6$ & $9.6 \mathrm{e} 6$ & $1.3 \mathrm{e} 7$ & $1.3 \mathrm{e} 7$ & $3 / 15$ \\
\hline CMA & & & & & & & & $\mathrm{CMA}$ & $1.9(1)$ & & $\infty$ & $\infty$ & $\infty$ & $\infty 1$ e 6 & $0 / 15$ \\
\hline $\mathrm{mir}$ & & 7.5( & 8.2( & 8 & & & & $\mathrm{mir}$ & $2.2(2)$ & & $\infty$ & $\infty$ & $\infty$ & & $0 / 15$ \\
\hline act & $8.7(6)$ & $7.2(6)$ & $\mathbf{7 . 9}(6)$ & $\mathbf{8 . 5}(6)$ & $3.7(2)$ & $3.7(2)$ & $15 / 15$ & act & $1.5(2)$ & $13(16)$ & $\infty$ & $\infty$ & $\infty$ & $\infty 1 e 6$ & $0 / 15$ \\
\hline
\end{tabular}

Table 1: Expected running time (ERT in number of function evaluations) divided by the respective best ERT measured during BBOB-2009 (given in the respective first row) for different $\Delta f$ values in dimension 5 . The central $80 \%$ range divided by two is given in braces. The median number of conducted function evaluations is additionally given in italics, if $\operatorname{ERT}\left(10^{-7}\right)=\infty$. \#succ is the number of trials that reached the final target $f_{\text {opt }}+10^{-8}$. Best results are printed in bold. 


\begin{tabular}{|c|c|c|c|c|c|c|c|c|c|c|c|c|c|c|c|}
\hline$\Delta f_{\text {opt }}$ & $1 \mathrm{e} 1$ & $1 \mathrm{e} 0$ & $1 \mathrm{e}-1$ & $1 \mathrm{e}-3$ & $1 e-5$ & $1 \mathrm{e}-7$ & \#succ & $\Delta f_{\text {opt }}$ & $1 \mathrm{e} 1$ & $1 \mathrm{e} 0$ & $1 \mathrm{e}-1$ & $1 \mathrm{e}-3$ & $1 e-5$ & $1 \mathrm{e}-7$ & \#succ \\
\hline f1 & 43 & 43 & 43 & 43 & 43 & 43 & $15 / 15$ & f13 & 652 & 2021 & 2751 & 18749 & 24455 & 30201 & $15 / 15$ \\
\hline CMA & $7.3(1)$ & $13(1)$ & $19(1)$ & $32(2)$ & $43(2)$ & $56(2)$ & $15 / 15$ & CMA & $2.5(0.4)$ & $5.1(4)$ & $7.5(6)$ & $1.7(1)$ & $1.9(0.9)$ & $2.0(1)$ & $15 / 15$ \\
\hline $\operatorname{mir}$ & $6.1(1)^{\star}$ & $10(1) \star 3$ & $14(2)^{\star 4}$ & $23(1)^{\star 4}$ & $32(1)^{\star 4}$ & $41(2)^{\star 4}$ & $15 / 15$ & mir & $3.1(4)$ & $3.2(4)$ & $6.2(4)$ & $1.7(1)$ & $2.4(0.9)$ & $2.4(0.7)$ & $15 / 15$ \\
\hline act & $7.8(1$ & $14(2)$ & $20(2)$ & $32(2)$ & $45(3)$ & $58(3)$ & $15 / 15$ & act & $2.4(0.3)$ & $3.5(3)$ & $4.5(3)$ & $1.1(0.8)$ & $1.2(0.7)$ & $1.5(1.0)$ & $15 / 15$ \\
\hline$\Delta f_{\text {opt }}$ & $1 \mathrm{e} 1$ & $1 \mathrm{e} 0$ & $1 \mathrm{e}-1$ & $1 \mathrm{e}-3$ & $1 \mathrm{e}-5$ & $1 \mathrm{e}-7$ & \#succ & $\Delta f_{\text {opt }}$ & $1 \mathrm{e} 1$ & $1 \mathrm{e} 0$ & $1 \mathrm{e}-1$ & $1 \mathrm{e}-3$ & $1 e-5$ & $1 \mathrm{e}-7$ & \#succ \\
\hline f2 & 385 & 386 & 387 & 390 & 391 & 393 & $15 / 15$ & f14 & 75 & 239 & 304 & 932 & 1648 & 15661 & $15 / 15$ \\
\hline CMA & $34(5)$ & $40(6)$ & $43(3)$ & $45(3)$ & $47(1)$ & $48(1)$ & $15 / 15$ & $\mathrm{CMA}$ & $4.5(2)$ & $2.9(0.6)$ & $3.7(0.5)$ & $4.1(0.4)$ & $6.1(0.5)$ & $1.2(0.1)$ & $15 / 15$ \\
\hline $\operatorname{mir}$ & $34(6)$ & $39(6)$ & $42(5)$ & $45(2)$ & $47(2)$ & $48(2)$ & $15 / 15$ & $\operatorname{mir}$ & $2.9(1)$ & $2.3(0.4)$ & $2.8(0.3) \star 2$ & $3.7(0.4)$ & $6.3(0.6)$ & $1.2(0.1)$ & $15 / 15$ \\
\hline act & $23(3) \star 3$ & $27(3) \star 3$ & $29(3)^{\star 4}$ & $31(2) \star 4$ & $32(2) \star 4$ & $34(2) \star 4$ & $15 / 15$ & act & $3.8(1)$ & $2.7(0.3)$ & $3.5(0.5)$ & $3.1(0.2) \star 3$ & $\mathbf{3 . 9}(0.2)^{\star 4}$ & $\mathbf{0 . 6 9}(0.0)_{\downarrow}^{\star 4} 4$ & $415 / 15$ \\
\hline$\Delta f_{\text {opt }}$ & $1 \mathrm{e} 1$ & $1 \mathrm{e} 0$ & $1 \mathrm{e}-1$ & $1 \mathrm{e}-3$ & $1 \mathrm{e}-5$ & $1 \mathrm{e}-7$ & \#succ & $\Delta f_{\text {opt }}$ & $\mid 1 \mathrm{e} 1$ & $1 \mathrm{e} 0$ & $1 \mathrm{e}-1$ & $1 e-3$ & $1 e-5$ & $1 \mathrm{e}-7$ & \#succ \\
\hline f3 & 5066 & 7626 & 7635 & 7643 & 7646 & 7651 & $15 / 15$ & $\frac{J_{\mathrm{opt}}}{\mathrm{f15}}$ & 30378 & $1.5 \mathrm{e} 5$ & $3.1 \mathrm{e} 5$ & $3.2 \mathrm{e} 5$ & $4.5 \mathrm{e} 5$ & $4.6 \mathrm{e} 5$ & $15 / 15$ \\
\hline CMA & $13(9)$ & $\infty$ & $\infty$ & $\infty$ & $\infty$ & $\infty 3 e 6$ & $0 / 15$ & CMA & $0.98(0.7)$ & $0.98(0.4)$ & $0.76(0.2)$ & $0.77(0.2)$ & $0.57(0.2)_{\downarrow}$ & $0.58(0.2) \downarrow$ & $15 / 15$ \\
\hline $\operatorname{mir}$ & $\begin{array}{l}8.5(6) \\
8.7(7)\end{array}$ & $\begin{array}{l}\infty \\
\infty\end{array}$ & $\begin{array}{l}\infty \\
\infty\end{array}$ & $\begin{array}{l}\infty \\
\infty\end{array}$ & $\begin{array}{l}\infty \\
\infty\end{array}$ & $\begin{array}{l}\infty 3 e 6 \\
\infty 3 e 6\end{array}$ & $\begin{array}{l}0 / 15 \\
0 / 15\end{array}$ & $\operatorname{mir}$ & $0.81(0.6)$ & $1.1(0.3)$ & $0.69(0.3)$ & $0.70(0.3)$ & $0.52(0.3) \downarrow 2$ & $20.53(0.3) \downarrow 2$ & $15 / 15$ \\
\hline$\Delta f_{\text {opt }}$ & $1 \mathrm{e} 1$ & $1 \mathrm{e} 0$ & $1 \mathrm{e}-1$ & $1 \mathrm{e}-3$ & $1 e-5$ & $1 \mathrm{e}-7$ & & act & $0.90(0.5)$ & $1.0(0.3)$ & $0.60(0.3)$ & $0.61(0.3)$ & $0.45(0.2) \downarrow 3$ & $3^{0.46(0.2}$ & $15 / 15$ \\
\hline $\mathrm{f} 4$ & 4722 & 7628 & 7666 & 7700 & 7758 & $1.4 \mathrm{e} 5$ & Fsuce & $\Delta f_{\text {opt }}$ & $1 \mathrm{e} 1$ & $1 \mathrm{e} 0$ & $1 \mathrm{e}-1$ & $1 \mathrm{e}-3$ & $1 e-5$ & $1 \mathrm{e}-7$ & \#succ \\
\hline CMA & $\infty$ & $\infty$ & $\infty$ & $\infty$ & $\infty$ & $\infty 3 e 6$ & $0 / 15$ & f16 & 1384 & 27265 & 77015 & $1.9 \mathrm{e} 5$ & $2.0 \mathrm{e} 5$ & $2.2 \mathrm{e} 5$ & $15 / 15$ \\
\hline ir & $\infty$ & $\infty$ & $\infty$ & $\infty$ & $\infty$ & $\infty 3 e 6$ & $0 / 15$ & $\mathrm{CMA}$ & & & $2(0.7)$ & & & & $15 / 15$ \\
\hline & $\infty$ & $\infty$ & $\infty$ & $\infty$ & $\infty$ & $\infty 3 e 6$ & $0 / 15$ & $\operatorname{mir}$ & $1.3(0.6)$ & $0.85(0.5)$ & $1.3(1)$ & $1.4(1)$ & $1.4(1)$ & 1.3 & $15 / 15$ \\
\hline$\Delta f_{\text {opt }}$ & $1 \mathrm{e} 1$ & $1 \mathrm{e} 0$ & $1 \mathrm{e}-1$ & $1 \mathrm{e}-3$ & $1 e-5$ & $1 \mathrm{e}-7$ & \#succ & act & $\mid 1.9(0.6)$ & $0.76(0.3)$ & $0.83(0.7)$ & $0.81(0.5)$ & $1.00(0.9)$ & $0.95(0.8)$ & $15 / 15$ \\
\hline f5 & 41 & 41 & 41 & 41 & 41 & 41 & $15 / 15$ & $\Delta f_{\text {opt }}$ & $1 \mathrm{e} 1$ & $1 \mathrm{e} 0$ & $1 \mathrm{e}-1$ & $1 e-3$ & $1 e-5$ & $1 \mathrm{e}-7$ & \#succ \\
\hline $\mathrm{CMA}$ & $4.9(1)$ & $5.7(0.9)$ & $5.9(1)$ & $5.9(1)$ & $5.9(1)$ & $5.9(1)$ & $15 / 15$ & f17 & 63 & 1030 & 4005 & 30677 & 56288 & 80472 & $15 / 15$ \\
\hline ir & 4.4(1) & $\mathbf{5 . 4}(1)$ & & & & & & $\mathrm{CMA}$ & & $1.00(0$. & & $0.81(0.3)$ & & & $15 / 15$ \\
\hline act & $5.5(1)$ & $6.5(2)$ & $6.6(2)$ & $6.6(2)$ & $6.6(2)$ & $6.6(2)$ & $15 / 15$ & $\operatorname{mir}$ & $2.2(0.5)$ & $0.82(0.3)$ & $1.4(1)$ & $\mathbf{0 . 5 9}(0.3) \downarrow 2$ & $2^{0.82(C}$ & $0.92(0.1)$ & $15 / 15$ \\
\hline$\Delta f_{\text {opt }}$ & $1 \mathrm{e} 1$ & $1 \mathrm{e} 0$ & $1 \mathrm{e}-1$ & $1 \mathrm{e}-3$ & $1 e-5$ & $1 \mathrm{e}-7$ & \#succ & act & $2.3(1)$ & $0.87(0.2)$ & $0.52(0.2)$ & $0.70(0.3)$ & $0.80(0.4)$ & $0.92(0.2)$ & $\mid 15 / 15$ \\
\hline $\mathrm{f6}$ & 1296 & & & & & 8409 & $15 / 15$ & $\Delta f_{\mathrm{opt}}$ & $1 \mathrm{e} 1$ & $1 \mathrm{e} 0$ & $1 \mathrm{e}-1$ & $1 \mathrm{e}-3$ & $1 e-5$ & $1 \mathrm{e}-7$ & \#succ \\
\hline CMA & 1.7 & & .1) & & .1) & & $15 / 15$ & f18 & 621 & 3972 & 19561 & 67569 & $1.3 \mathrm{e} 5$ & $1.5 \mathrm{e} 5$ & $15 / 15$ \\
\hline in & 1.7( & & & & & & $15 / 15$ & $\mathrm{CMA}$ & $0.96(0.2)$ & $(0.4)$ & 0.8 & $0.3)$ & & & $15 / 15$ \\
\hline act & $1.6(0.3)$ & $1.3(0.2)$ & $1.1(0.1)$ & $1.1(0.1)$ & $1.1(0.1)$ & $1.1(0$. & $15 / 15$ & $\operatorname{mir}$ & & & & & & & $15 / 15$ \\
\hline$\Delta f_{\text {opt }}$ & $1 \mathrm{e} 1$ & $1 \mathrm{e} 0$ & $1 \mathrm{e}-1$ & $1 \mathrm{e}-3$ & $1 e-5$ & $1 e-7$ & \#succ & act & $0.96(c$ & $0.96(2)$ & $0.96(0.9)$ & $0.79(0.3)$ & $\mathbf{0 . 8 5}(0$. & $\mathbf{0 . 8 7}(0$. & $15 / 15$ \\
\hline$f 7$ & 1351 & 4 & 9503 & 16524 & 16524 & 16969 & $15 / 15$ & $\Delta f_{\text {opt }}$ & $1 \mathrm{e} 1$ & $1 \mathrm{e} 0$ & $1 \mathrm{e}-1$ & $1 \mathrm{e}-3$ & $1 \mathrm{e}-5$ & $1 \mathrm{e}-7$ & \#succ \\
\hline $\mathrm{CMA}$ & $1.7(1)$ & & & & & 1 & $15 / 15$ & f19 & 1 & 1 & $3.4 \mathrm{e} 5$ & $6.2 \mathrm{e} 6$ & $6.7 \mathrm{e} 6$ & $6.7 \mathrm{e} 6$ & $15 / 15$ \\
\hline & $1.7(1)$ & & & 1.7( & 1.7 & & $15 / 15$ & CMA & 170 & $3.1 \mathrm{e}$ & 4) 2.0 ( & 0.9 & & & $5 / 15$ \\
\hline act & $1.0(1.0)$ & $\mathbf{2 . 3}(1.0)$ & $1.7(0.7)^{\star}$ & $1.1(0.4)^{\star}$ & $1.1(0.4)^{\star}$ & $1.0(0.4)^{\star}$ & $15 / 15$ & $\operatorname{mir}$ & $134(58)$ & & & & & & $3 / 15$ \\
\hline$\Delta f_{\text {opt }}$ & $1 \mathrm{e} 1$ & $1 \mathrm{e} 0$ & $1 \mathrm{e}-1$ & $1 \mathrm{e}-3$ & $1 e-5$ & $1 e-7$ & \#succ & & $156(72)$ & $7.7 \mathrm{e}$ & 4) $2.5(4)$ & $\mathbf{0 . 7 3}(0.6)$ & $0.88(0.9)$ & $0.88(0.8)$ & $8 / 15$ \\
\hline f8 & 2039 & 3871 & 4040 & 4219 & 4371 & 4484 & $15 / 15$ & $\Delta f_{\mathrm{opt}}$ & $1 \mathrm{e} 1$ & $1 \mathrm{e} 0$ & $1 \mathrm{e}-1$ & $1 \mathrm{e}-3$ & $1 e-5$ & $1 e-7$ & \#succ \\
\hline CMA & $3.7(0.6)$ & $4(0.3)$ & $7(0.3)$ & $4.9(0.3)$ & $4.9(0.3)$ & $5.0(0$ & $15 / 15$ & f20 & 82 & 46150 & $3.1 \mathrm{e} 6$ & $5.5 \mathrm{e} 6$ & $5.6 \mathrm{e} 6$ & $5.6 \mathrm{e} 6$ & $14 / 15$ \\
\hline hir & $3.9(0.7)$ & $5.0(4)$ & $5.3(4)$ & $5.4(3)$ & $5.4(3)$ & $5.4(3)$ & $15 / 15$ & CMA & $4.8(1)$ & $5.4(2)$ & $(0.4)$ & & & & $6 / 15$ \\
\hline act & 3.6(0.7) & $3.5(0.6)^{\star 2}$ & $3.8(0.6)^{\star 2}$ & $4.0(0.6)^{\star 2}$ & $4.0(0.6)^{\star 2}$ & $4.0(0.6)^{\star 2}$ & $15 / 15$ & $\operatorname{mir}$ & $3.4(0.7)^{\star 2}$ & $5.4(3)$ & & & 2.0 & $3.3(4)$ & $3 / 15$ \\
\hline$\Delta f_{\text {opt }}$ & $1 \mathrm{e} 1$ & $1 \mathrm{e} 0$ & $1 \mathrm{e}-1$ & $1 \mathrm{e}-3$ & $1 e-5$ & $1 \mathrm{e}-7$ & \#succ & & & & & & & & $5 / 15$ \\
\hline $\mathrm{fg}$ & 1716 & & & & & & $\frac{15 / 15}{15}$ & $\Delta f_{\text {opt }}$ & $1 \mathrm{e} 1$ & $1 \mathrm{e} 0$ & $1 \mathrm{e}-1$ & $1 \mathrm{e}-3$ & $1 e-5$ & $1 \mathrm{e}-7$ & \#succ \\
\hline MA & $4.7(0.9)$ & & $4(0.6$ & 5 & 5.6( & 5.6 & $15 / 15$ & f21 & 56 & 6541 & 14103 & 146 & 155 & & $15 / 15$ \\
\hline mir & 4.11 & $5.4(0.7)$ & $5.7(0.6)$ & $5.8(0.6)$ & $5.8(0.6)$ & $5.8(0.6)$ & $15 / 15$ & $\mathrm{CMA}$ & & & & & & & $9 / 15$ \\
\hline act & $3.9(0.7)$ & $4.1(0.4)^{\star 2}$ & $4.4(0.4)^{\star 2}$ & $4.5(0.4)^{\star 2}$ & $4.5(0.4)^{\star 2}$ & $4.5(0.4) \star 2$ & $15 / 15$ & $\operatorname{mir}$ & $3.5(4)$ & $109(177)$ & & $77(113)$ & & & \\
\hline$\Delta f_{\text {opt }}$ & $1 \mathrm{e} 1$ & $1 \mathrm{e} 0$ & $1 \mathrm{e}-1$ & $1 \mathrm{e}-3$ & $1 e-5$ & $1 \mathrm{e}-7$ & \#succ & act & 3.2(4) & $\mathbf{9 5}(175)$ & $77(105)$ & $74(87)$ & $70(85)$ & $62(97)$ & $8 / 15$ \\
\hline f10 & 7413 & & 10735 & & & & $15 / 15$ & $\Delta f_{\text {opt }}$ & $1 \mathrm{e} 1$ & $1 \mathrm{e} 0$ & $1 \mathrm{e}-1$ & $1 \mathrm{e}-3$ & $1 \mathrm{e}-5$ & $1 \mathrm{e}-7$ & \#succ \\
\hline CMA & $1.8(0.3$ & & & & & & $15 / 15$ & f22 & 46 & 5580 & 23491 & 24948 & 26847 & $1.3 \mathrm{e} 5$ & $12 / 15$ \\
\hline $\operatorname{mir}$ & $1.8(0.2)$ & $1.8(0.2)$ & $1.6(0.1)$ & $1.2(0.0)$ & $1.1(0.0)$ & $1.1(0.0)$ & $15 / 15$ & CMA & & & $\infty$ & & $\infty$ & & $0 / 15$ \\
\hline act & $1.2(0.2)^{\star 3}$ & $1.2(0.2)^{\star 4}$ & $1.0(0.1)^{\star 4}$ & $\mathbf{0 . 8 2}(0.0)_{\downarrow 4}^{\star 4}$ & ${ }_{4}^{4} \mathbf{0 . 7 5}(0.0)_{\downarrow}^{\star 4}$ & ${ }_{4}^{4} \mathbf{0 . 7 6}(0.0)_{\downarrow}^{\star}$ & $15 / 15$ & $\mathrm{~m}$ & $\mid \begin{array}{l}\mathbf{7 . O}(12) \\
10(13)\end{array}$ & $\begin{array}{l}188(2 \\
232(3\end{array}$ & $\infty$ & $\begin{array}{l}\infty \\
\infty\end{array}$ & & $\begin{array}{l}\infty 1 \text { e } 6 \\
\infty 1 \text { e } 6\end{array}$ & $\begin{array}{l}0 / 15 \\
0 / 15\end{array}$ \\
\hline$\Delta f_{\text {opt }}$ & $1 \mathrm{e} 1$ & $1 \mathrm{e} 0$ & $1 \mathrm{e}-1$ & $1 \mathrm{e}-3$ & $1 \mathrm{e}-5$ & $1 \mathrm{e}-7$ & \#succ & $\Delta f_{\text {opt }}$ & $1 \mathrm{e} 1$ & $1 \mathrm{e} 0$ & $1 e-1$ & $1 \mathrm{e}-3$ & $1 \mathrm{e}-5$ & $1 \mathrm{e}-7$ & \#succ \\
\hline f11 & 1002 & 2228 & 278 & 762 & 12285 & 14831 & $15 / 15$ & $\frac{f o p t}{f 23}$ & 3.2 & 1614 & 67457 & $4.9 \mathrm{e} 5$ & $8.1 \mathrm{e} 5$ & $8.4 \mathrm{e} 5$ & $15 / 15$ \\
\hline CMA & $10(1.0)$ & $5.1(0.3)$ & & & & & $15 / 15$ & & $3.4(4)$ & & & $\infty$ & $\infty$ & & $0 / 15$ \\
\hline mir & $11(0.7)$ & $5.4(0.4)$ & $2.0(0.1)$ & $1.4(0.1)$ & $1.2(0.1)$ & $1.1(0.0)$ & $15 / 15$ & mir & $3.9(4)$ & $1.1 \mathrm{e} 4(1 \mathrm{e} 4)$ & $577(664)$ & $\infty$ & $\infty$ & $\infty 3 e 6$ & $0 / 15$ \\
\hline act & $4.5(0.2)^{\star 4}$ & $2.2(0.1)^{\star 4}$ & ${ }^{4} 0.86(0.0)^{\star}$ & ${ }^{4} \mathbf{0 . 6 3}(0.0)_{\downarrow}^{\star}$ & ${ }_{4}^{4} 0.55(0.0)$ & ${ }_{4}^{4} \mathbf{0 . 5 0}(0.0)$ & $15 / 15$ & act & $6.5(5)$ & $1.1 \mathrm{e} 4(1 \mathrm{e} 4)$ & $556(593)$ & $\infty$ & $\infty$ & $\infty 3 e 6$ & $0 / 15$ \\
\hline$\Delta f_{\text {opt }}$ & $1 \mathrm{e} 1$ & $1 \mathrm{e} 0$ & $1 \mathrm{e}-1$ & $1 \mathrm{e}-3$ & $1 \mathrm{e}-5$ & $1 \mathrm{e}-7$ & \#succ & $\Delta f_{\mathrm{opt}}$ & $1 \mathrm{e} 1$ & $1 \mathrm{e} 0$ & $1 \mathrm{e}-1$ & $1 \mathrm{e}-3$ & $1 \mathrm{e}-5$ & $1 \mathrm{e}-7$ & \#succ \\
\hline f12 & & & & & & & $15 / 15$ & f24 & $1.3 \mathrm{e} 6$ & $7.5 \mathrm{e} 6$ & $5.2 \mathrm{e} 7$ & $5.2 \mathrm{e} 7$ & $5.2 \mathrm{e} 7$ & 5.2 & $3 / 15$ \\
\hline & & & & & & & & & $\infty$ & $\infty$ & $\infty$ & $\infty$ & $\infty$ & & \\
\hline $\operatorname{mir}$ & $3.2(4)$ & $4.1(5)$ & $4.8(5)$ & .6(3) & $2.0(1)$ & $2.1(1$ & $15 / 15$ & $\operatorname{mir}$ & $12(15)$ & $\mathbf{3 . 6}(4)$ & $\infty$ & $\infty$ & $\infty$ & $\infty 4 e 6$ & $0 / 15$ \\
\hline act & $2.4(0.2)$ & $3.4(2)$ & $3.4(2)$ & $3.4(1)$ & $1.4(0.5)$ & $1.5(0.5)$ & $15 / 15$ & act & $42(48)$ & $\infty$ & $\infty$ & $\infty$ & $\infty$ & $\infty 4 e 6$ & $0 / 15$ \\
\hline
\end{tabular}

Table 2: Expected running time (ERT in number of function evaluations) divided by the respective best ERT measured during BBOB-2009 (given in the respective first row) for different $\Delta f$ values in dimension 20 . The central $\mathbf{8 0 \%}$ range divided by two is given in braces. The median number of conducted function evaluations is additionally given in italics, if $\operatorname{ERT}\left(10^{-7}\right)=\infty$. \#succ is the number of trials that reached the final target $f_{\text {opt }}+10^{-8}$. Best results are printed in bold. 